\title{
INVESTIGACIÓN
}

Recibido: 25/04/2018 --- Aceptado: 04/04/2019 --- Publicado: 15/06/2019

\section{EXPERIENCIAS DE TRABAJO COOPERATIVO EN LA EDUCACIÓN SUPERIOR. PERCEPCIONES SOBRE SU CONTRIBUCIÓN AL DESARROLLO DE LA COMPETENCIA SOCIAL}

\section{Experiences of cooperative work in higher education. Perceptions about its contribution to the development of social competence}

(iD) (8) $\mathbb{R}^{\text {I }}$ Francisco José Sánchez Marín: Universidad Católica San Antonio de Murcia. España.

fjsanchez235@ucam.edu

(D) (8) $\mathbb{R}^{R}$ María Concepción Parra-Meroño1: Universidad Católica San Antonio de Murcia. España.

mcparra@ucam.edu

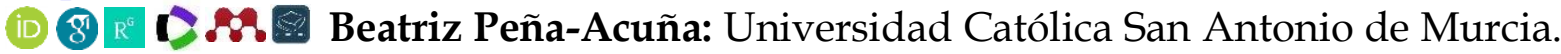

España.

bpena@ucam.edu

\section{RESUMEN}

El Espacio Europeo de Educación Superior (EEES) exige el desarrollo de competencias clave para dar respuesta a las demandas de la sociedad y del mercado de trabajo. La Competencia Social incluye destrezas fundamentales para las relaciones sociales de calidad en el ámbito personal y profesional. Se identifica el Aprendizaje Cooperativo como método apropiado para lograr tal fin.

En este artículo se presenta la experiencia de una investigación cualitativa durante los cursos 2009-2015 en la que se vertebra la investigación acción participativa como una técnica adecuada, puesto que investiga a la muestra, se diseña una metodología docente en base a las carencias y se vuelve a investigar la eficacia del aprendizaje debido a la metodología. Exponemos un caso en el que la investigación acción participativa ha sido aplicada al campo de la Educación universitaria en la que se asegura más la adecuación a la diversidad precisamente por la adecuación que otorga al aula investigada.

Además, se exponen los resultados del análisis de esta investigación e innovación, en concreto, la percepción expresada por los alumnos acerca del grado en que el trabajo en equipo cooperativo contribuye al desarrollo de su Competencia Social tras participar en

\footnotetext{
${ }^{1}$ María Concepción Parra Meroño. Doctora en Administración y Dirección de empresas: Marketing y Organización. Es profesora en el Grado en ADE y en el Master en Marketing y Comunicación y en el Master MBA de la UCAM.
} 
Sánchez-Marín, F. J., Parra-Meroño, M. C., y Peña-Acuña, B. Experiencias

de trabajo cooperativo en la educación superior. Percepciones sobre su contribución al desarrollo de la competencia social

una experiencia de Aprendizaje Cooperativo. Se diseña un tipo de investigación aplicada, desde una perspectiva cualitativa, no experimental, en el ámbito de la evaluación de resultados para la toma de decisiones. Participan 126 estudiantes de Grado de primer y cuarto curso. A través de un cuestionario tipo Likert, se recoge su percepción sobre competencias emergentes al desempeño de la Competencia Social: Empatía, Consenso o Participación Democrática y Asertividad. Este estudio aporta novedad en tanto que explora sobre una muestra de estudiantes con perfiles diversos, tomando como punto de partida de manera integrada diversas variables y sobre la percepción que los propios estudiantes expresan de manera explícita. Se confirma que los estudiantes perciben que, a través del Aprendizaje Cooperativo, se desarrolla su Competencia Social. Las habilidades mejor valoradas son las que se relacionan con el ámbito cognitivo y se relega a un segundo plano otras de carácter emocional, axiológico y paradigmático.

PALABRAS CLAVE: competencia social - investigación acción - cooperación educativa - inteligencia emocional - competencia profesional - comunicación interpersonal.

\section{ABSTRACT}

The European Higher Education Area (EHEA) requires the development of key competences to respond to the demands of society and the labor market. Social Competence includes fundamental skills for quality social relationships in the personal and professional sphere. Cooperative Learning is identified as an appropriate method to achieve this goal.

This article presents the experience of qualitative research during the 2009-2015 courses in which participatory action research is structured as an appropriate technique, since it investigates the sample, a teaching methodology is designed based on the shortcomings and returns to investigate the effectiveness of learning due to the methodology. We present a case in which participatory action research has been applied to the field of university education in which the adaptation to diversity is more precisely assured by the adequacy that it gives to the classroom under investigation.

Besides presents the results of the analysis of the perception expressed by the students about the degree to which cooperative teamwork contributes to the development of their Social Competence after participating in a Cooperative Learning experience. A type of applied research is designed, from a qualitative perspective, not experimental, in the field of evaluation of results for decision making. 126 First and Fourth Grade students participate. Through a Likert-type questionnaire, their perception of emerging competences is gathered in the performance of the Social Competence: Empathy, Consensus or Democratic Participation and Assertiveness. This study provides novelty in that it explores a sample of students with diverse profiles, taking as a starting point in an integrated way various variables and on the perception that the students themselves express explicitly. It is confirmed that the students perceive that, through Cooperative Learning, their Social Competence is developed. The best valued skills are those related to 
Sánchez-Marín, F. J., Parra-Meroño, M. C., y Peña-Acuña, B. Experiencias

de trabajo cooperativo en la educación superior. Percepciones sobre su contribución al desarrollo de la competencia social

the cognitive field and others of an emotional, axiological and paradigmatic nature are relegated to the background.

KEY WORDS: social competence - action research - educational cooperation - emotional intelligence - professional competence - interpersonal communication.

\section{EXPERIÊNCIAS DE TRABALHO COOPERATIVO NA EDUCAÇÃO SUPERIOR. PERCEPÇÕES SOBRE SUA CONTRIBUIÇÃO AO DESENVOLVIMENTO DA ATRIBUIÇÃO SOCIAL}

\section{RESUME}

O espaço europeu de Educação Superior (EEES) exige o desenvolvimento de atribuições chave para dar respostas as demandas da sociedade e do mercado de trabalho. A Atribuição Social inclui destrezas fundamentais para as relações sociais de qualidade no âmbito pessoal e profissional. Identifica-se o Aprendizagem Cooperativo como método apropriado para lograr essa finalidade. Neste artigo apresenta-se a experiência de uma investigação qualitativa durante os cursos dos anos 2009-2015 na qual estrutura a investigação ação participativa como técnica adequada, investigando a amostra e desenhando uma metodologia docente com base as carências e eficácia da aprendizagem devido a essa metodologia.

Expomos um caso no qual a investigação ação participativa foi aplicada ao campo da Educação universitária na qual se assegura mais a adequação do que a diversidade precisamente pela adequação que outorga a aula investigada.

Ademais, expõe-se os resultados da analises desta investigação e inovação, em concreto, a percepção expressada pelos alunos aproxima. Do grau no qual o trabalho em equipe cooperativo contribui ao desenvolvimento de sua atribuição Social depois de participar em uma experiência de Aprendizagem Cooperativa.

Se planeja um tipo de investigação aplicada, desde uma perspectiva qualitativa, não experimentada, no âmbito da valoração de resultados para a toma de decisões.

Participam 126 estudantes do primeiro e quarto ano do curso universitário. Através de um questionário tipo Likert, são coletadas suas percepções sobre atribuições emergentes ao desempenho da Atribuição Social: Empatia, consenso ou participação Democrática e Assertividade. Este estudo aporta novidades já que explora sobre uma amostra de estudantes com diversos perfis, tomando como ponto de partida de maneira integrada diversas variáveis e sobre a percepção que os próprios estudantes expressam de maneira explicita. Confirmando que os estudantes notam que, através da Aprendizagem Cooperativa, desenvolvem sua Atribuição Social. As habilidades mais valorizadas são as que se relacionam com o âmbito cognitivo e se relega a um segundo plano outras de caráter emocional, axiológico paradigmático.

PALAVRAS CHAVE: atribuição social - investigação ação - cooperação educativa inteligência emocional - atribuição profissional - interpessoal. 
Sánchez-Marín, F. J., Parra-Meroño, M. C., y Peña-Acuña, B. Experiencias

de trabajo cooperativo en la educación superior. Percepciones sobre su contribución al desarrollo de la competencia social

\section{Como citar el artículo:}

Sánchez-Marín, F. J., Parra-Meroño, M. C., y Peña-Acuña, B. (2019). Experiencias de trabajo cooperativo en la educación superior. Percepciones sobre su contribución al desarrollo de la competencia social. [Experiences of cooperative work in higher education. Perceptions about its contribution to the development of social competence]. Vivat Academia. Revista de Comunicación, 147, 87-108. http://doi.org/10.15178/va.2019.147.87-108. Recuperado de http:// www.vivatacademia.net/index.php/vivat/article/view/1123

\section{INTRODUCCIÓN}

Esta disertación expone la aplicación de la investigación-acción participativa con carácter beneficioso para la innovación educativa, en concreto, en la Educación universitaria. Durante los cursos 2009-2012 se detectó por parte del profesorado la carencia de habilidades de trabajo en equipo por parte de los discentes. Además, esta cuestión se veía como una necesidad urgente de cara a la formación profesional en consonancia con el marco de Bolonia. Esta realidad fue observada y comentada cada año en las tres reuniones de curso por parte del profesorado.

En el curso siguiente (2013-2014), varios profesores que también lo llevaban observando de forma participativa, deciden realizar un proyecto piloto mediante la investigación-acción participativa a un grupo de 50 alumnos que cursaban la asignatura de "Habilidades sociales" en el grado de Magisterio. Siguiendo a Peña (2014), se despliegan las siguientes herramientas. Primero, realiza un cuestionario previo a los alumnos en el que se formulan preguntas abiertas acerca de las habilidades de trabajo en equipo de forma general. Segundo, les impartió dos clases con nociones básicas de habilidades de trabajo en equipo. Tercero, siguió el trabajo de los equipos, realizó grupos de discusión sobre su desenvolvimiento, entrevistó a algunos alumnos y realizó una encuesta al final del curso para comprobar qué mejoras percibían los estudiantes sobre sus habilidades de trabajo en equipo y qué habilidades destacaban como más necesarias. De este estudio, sobresalieron tres variables y ciertas manifestaciones sobre ellas: la actitud de consenso, la asertividad y la empatía.

Después y gracias a esta investigación-acción participativa, un grupo multidisciplinar de 13 docentes e investigadores aplica un Proyecto de Innovación Docente Cooperativo con el fin de propiciar en los estudiantes el desarrollo de su Competencia Social, mediante el entrenamiento de destrezas comunicativas, utilizando estrategias de Aprendizaje Cooperativo. El proyecto fue financiado con duración de 12 meses (PMFI PID 14-14) durante el curso 2014-2015. Desde un principio se proyecta que primero es el profesorado el que debe aprender a trabajar en equipo. Para ello se crea una carpeta de materiales compartida en Google Drive. Además, se forman equipos de trabajo para 
Sánchez-Marín, F. J., Parra-Meroño, M. C., y Peña-Acuña, B. Experiencias de trabajo cooperativo en la educación superior. Percepciones sobre su contribución al desarrollo de la competencia social

crear los textos y protocolos de actuación del profesorado participante en el proyecto. De ese modo, los docentes realizan una investigación sobre estos conceptos del proyecto, se forman en ellos y los viven de forma experiencial al tener que trabajar en equipo.

Una vez se creó el protocolo, los textos y la secuenciación de sesiones y el profesorado estaba preparado, se aplicó el proyecto de innovación en varias asignaturas durante un semestre teniendo en cuenta que el alumnado tenía una carencia al respecto conocida por la investigación y experiencia previa.

Ya finalizada la metodología docente, para confirmar su pertinencia y seguir replicando la experiencia cooperativa, se solicitó a los estudiantes que expresaran mediante un cuestionario su percepción sobre el grado en que consideraban que el trabajo en equipo cooperativo desplegado había contribuido a desarrollar su Competencia Social. Los resultados se exponen en esta disertación con el fin de mostrar los resultados científicos de todo este proceso de profundización cualitativa.

El Espacio Europeo de Educación Superior (EEES) propone la formulación de los objetivos de enseñanza como resultados de aprendizaje, expresados como competencias y vinculadas a perfiles profesionales (Cano, 2008). Un nuevo modelo formativo orientado a mejorar la capacidad de respuesta a las demandas sociales y del mercado de trabajo, atender las rápidas transformaciones sociales (Román, Vecina, Usategui, del Valle y Venegas, 2015), la globalización (Trujillo y Sánchez, 2013), el impacto de las Tecnologías de la Información y la Comunicación (TIC) (Aguaded-Gómez, 2013), la administración del conocimiento (Rabadán y Pérez, 2012), así como la gestión de la diversidad (Más y Olmos, 2012).

Derivado del ámbito de la formación y la gestión profesional, el concepto de competencia se inserta en los últimos años en la educación en general (European Commission/EACEA/Eurydice, 2015) y adopta una posición central en la Educación Superior. El concepto de competencia ha sido sometido a numerosos intentos de definición desde diferentes disciplinas y ámbitos (Mulder, Weigel y Collings, 2008; Ortiz, Vicedo, González y Recino, 2015; Restrepo, 2014). Se consideran competencias básicas aquellas que tienen como finalidad la integración de las TIC y la comunicación, el impulso de la cultura tecnológica y las lenguas extranjeras, así como el espíritu emprendedor y las habilidades sociales (Ferrés, 2007). Sin embargo, no existe un consenso unánime acerca de lo que realmente son y cómo conviene entenderlas en el trabajo universitario, al margen de su vinculación directa con el mundo laboral.

En este contexto, un grupo de universidades europeas impulsa el Proyecto Tuning Europa con el objetivo de armonizar las estructuras docentes en la Educación Superior. Así, se determinan puntos de referencia para las competencias genéricas y específicas de 
Sánchez-Marín, F. J., Parra-Meroño, M. C., y Peña-Acuña, B. Experiencias de trabajo cooperativo en la educación superior. Percepciones sobre su contribución al desarrollo de la competencia social

cada disciplina en una serie de ámbitos temáticos: estudios empresariales, ciencias de la educación, geología, historia, matemáticas, física y química, entre otras. En este estudio de tipo evaluativo reformulamos algunas competencias genéricas relacionadas con la comunicación social, relaciones interpersonales y sociales, asociadas al constructo Inteligencia Interpersonal o Competencia Social.

El constructo Inteligencia Interpersonal es un subtipo de Inteligencia que se encuentra entre las ocho que propone Gardner (1985) en su Teoría de las Inteligencias Múltiples, en la que más tarde profundizaron Salovey y Mayer (1990), hasta desarrollar el constructo Inteligencia Emocional. Éste concepto alcanzaría su máximo grado de difusión e impacto con la obra de Goleman (2006), quien amplía el marco de referencia más allá de lo unipersonal, para adentrarse en la psicología de las relaciones que se mantienen con los demás; Inteligencia Social. Según Gardner (2005, p. 47), "la inteligencia interpersonal se construye a partir de una capacidad nuclear para sentir distinciones entre los demás: en particular, contrastes en sus estados de ánimo, temperamentos, motivaciones e intenciones". Podríamos decir entonces que, una persona con un adecuado desarrollo de su inteligencia interpersonal, sería capaz de diferenciar a las personas con las que se relaciona en función de lo que expresan (estados de ánimo, temperamentos, motivaciones e intenciones).

Por su parte, Bisquerra y Pérez (2007, p. 72) revisan y actualizan el modelo en torno a las competencias emocionales y asimilan Inteligencia interpersonal a Competencia Social como "capacidad para mantener buenas relaciones con otras personas. Esto implica dominar las destrezas sociales: capacidad para la comunicación efectiva, respeto, actitudes pro-sociales, asertividad, etc.". La importancia de la Competencia Social deriva, entonces, del continuo contacto que mantenemos con nuestros iguales desde nuestro nacimiento. Podemos afirmar, incluso, que estamos genéticamente programados para relacionarnos con otras personas. Aunque esta predisposición no garantice la calidad de las mismas. Decety, Norman, Berntson y Cacioppo (2012) afirman que las conductas relacionadas con la Empatía, por ejemplo, han colaborado en los procesos homeostáticos más primitivos que intervienen en los sistemas de recompensa y dolor con el fin de facilitar diversos procesos de apego social.

Estas emociones compartidas pueden facilitar la preocupación empática que promueve conductas pro-sociales y el altruismo. La cooperación, por ejemplo, es un aspecto fundamental de todos los sistemas biológicos, desde las bacterias hasta los primates, incluidos los seres humanos (Decety, Bartal, Uzefovsky y Knafo-Noam, 2016). Por tanto, en todas las etapas educativas, incluida la Educación Superior, es preciso generar estructuras de aprendizaje que desarrollen la cooperación como aspecto esencial para el desarrollo de la Competencia Social. Consideramos que las estructuras basadas en el Aprendizaje Cooperativo son de las estrategias educativas más adecuadas para lograrlo. García y Troyano (2010, p. 8) avalan esta convicción al afirmar que: “el 
Sánchez-Marín, F. J., Parra-Meroño, M. C., y Peña-Acuña, B. Experiencias

de trabajo cooperativo en la educación superior. Percepciones sobre su contribución al desarrollo de la competencia social

aprendizaje cooperativo procura la construcción colectiva del conocimiento y el desarrollo de habilidades mixtas -aprendizaje y desarrollo personal y social-, donde cada miembro del grupo es responsable de su aprendizaje y del de los restantes miembros del grupo". Con mayor precisión se puede plantear como sigue:

La cooperación consiste en trabajar juntos para alcanzar objetivos comunes. En una situación cooperativa, los individuos procuran obtener resultados que sean beneficiosos para ellos mismos y para todos los demás miembros del grupo. El aprendizaje cooperativo es el empleo didáctico de grupos reducidos en los que los estudiantes trabajan juntos para maximizar su propio aprendizaje y el de los demás (Johnson, Johnson y Holubec, 1999, p. 5).

Para que realmente se produzca cooperación, se plantean cinco elementos esenciales (Johnson, Johnson y Holubec, 1999):

a) Interdependencia positiva: Cada miembro tiene claro que su esfuerzo no sólo lo benefician a él, sino también a los demás.

b) Responsabilidad individual y grupal: el grupo y cada miembro asume la responsabilidad de alcanzar los objetivos.

c) Interacción estimuladora: Cada participante promueve el éxito de los demás, compartiendo los recursos y prestando unos a otros ayuda, respaldo, aliento y ánimo por su empeño en aprender.

d) Aprendizaje de prácticas interpersonales y grupales: dirección, toma de decisiones, confianza, comunicación y gestión de conflictos.

e) Evaluación grupal: Los propios componentes del grupo analizan en qué medida en están alcanzando sus metas.

La literatura actual evidencia que el aprendizaje en equipo cooperativo mejora las habilidades sociales básicas para ser eficaz en otras situaciones similares (Del Barco, Felipe, Mendo y Iglesias, 2015), la comunicación transactiva y elaboración de ideas propias y compartidas (Jurkowski y Hanze, 2015), las actitudes hacia el aprendizaje (Gil, 2015) y las competencias globales (Cebrián-de la Serna, Serrano-Angulo y Ruiz-Torres, 2014), específicas y transversales (Oricain, 2015). Resulta evidente, por tanto, la superioridad del aprendizaje cooperativo sobre el individual en el rendimiento de estudiantes universitarios y la potencia y confianza en el equipo de trabajo (Camilli, 2015; Del Barco, Mendo, Felipe, Polo y Fajardo, 2015).

\section{OBJETIVOS}

El objetivo general que se pretende con este trabajo es demostrar que el trabajo cooperativo contribuye al desarrollo de la competencia social, la cual incluye una serie de competencias fundamentales para las relaciones sociales de calidad en el ámbito 
Sánchez-Marín, F. J., Parra-Meroño, M. C., y Peña-Acuña, B. Experiencias

de trabajo cooperativo en la educación superior. Percepciones sobre su contribución al desarrollo de la competencia social

personal y profesional, tal y como exige el EEES para dar respuesta a las demandas de la sociedad y del mercado laboral.

\section{METODOLOGÍA}

El Proyecto de Innovación Docente Cooperativo implementado se focalizó en 4 asignaturas impartidas por profesores integrantes del mismo con el fin de asegurar su viabilidad y rigor en los planteamientos. Se desarrolló durante el curso académico 201516, con una duración total de 6 meses, estructurados en sesiones semanales de 2 horas de duración. El tipo de tareas realizadas fueron eminentemente prácticas: elaboración de protocolos de actividad, diseño de sistemas de procesos y rutinas o confección de recursos y materiales didácticos. La ejecución de las tareas en las materias correspondientes consistió en convertir el contenido teórico en producciones prácticas de los mismos. El trazado metodológico común para todas ellas se inspiró en el modelo de Arnaiz y Linares (2010):

a) Explicación, por parte del profesor a los estudiantes, sobre aspectos clave del aprendizaje cooperativo: agrupamientos, reparto de tareas, modelo de interacción entre componentes de los grupos, tratamiento de los conflictos personales emergentes y recompensas.

b) Configuración de los equipos de trabajo conforme a los siguientes criterios: limitación del número de componentes (entre 4 y 6 estudiantes), estabilidad (mantenimiento de los equipos hasta finalizar todas las tareas) y heterogeneidad de perfiles. Este último criterio se justifica en que los alumnos con perfil heterogéneo tienen acceso a diversas perspectivas y métodos de resolución de problemas, por lo que se produce un mayor desequilibrio cognitivo (necesario para estimular el aprendizaje), promover un pensamiento más profundo, un mayor intercambio de explicaciones y una mayor tendencia a asumir puntos de vista diversos (Johnson, Johnson y Holubec, 1999). La selección de los alumnos, buscando la heterogeneidad, se realizó previa observación del profesor sobre indicadores como el estilo de trabajo, el nivel de competencia académica y en materias de conocimiento específicas o los rasgos de personalidad.

c) Distribución de tareas complementarias en los equipos, en función de las características del alumnado, la materia y la actividad, siendo necesario el trabajo de todos los grupos para finalizar la tarea global.

d) Elaboración del calendario de trabajo del profesor y estudiantes con indicación precisa de las sesiones de trabajo en el aula, así como materiales y recursos necesarios para realizar la actividad.

e) Seguimiento y supervisión de las tareas mediante el establecimiento de canales de comunicación fluida entre profesor y grupos de trabajo.

Para recopilar la percepción de los estudiantes, se diseñó un procedimiento de investigación aplicada (Gast y Ledford, 2014), desde una perspectiva cualitativa, no 
Sánchez-Marín, F. J., Parra-Meroño, M. C., y Peña-Acuña, B. Experiencias

de trabajo cooperativo en la educación superior. Percepciones sobre su contribución al desarrollo de la competencia social

experimental, enmarcada en el ámbito de la evaluación de resultados para la toma de decisiones y centrada en la valoración de la aceptación y satisfacción de la población objetivo (Mertens, 2014).

La muestra se compuso de estudiantes que participaron en el Proyecto de Innovación Docente Cooperativo aplicado; 126 estudiantes de primer y cuarto curso, en la modalidad de estudio presencial, de los Grados en Educación Infantil, Educación Primaria y Administración y Dirección de Empresas, así como del Ciclo de Formativo de Grado Superior de Formación Profesional de Educación Infantil, de una institución de Educación Superior de titularidad privada, con edades comprendidas entre 18 y 25 años y una representación del 84\% del género femenino y el 16\% del masculino (Tabla 1).

Tabla 1. Composición de la muestra.

\begin{tabular}{lcccc}
\hline \multicolumn{1}{c}{ Titulación } & Curso & $\begin{array}{c}\mathbf{N}^{\mathbf{0}} \text { de } \\
\text { estudiantes }\end{array}$ & Mujeres & Hombres \\
\hline $\begin{array}{l}\text { Técnico Superior en Educación } \\
\text { Infantil }\end{array}$ & Primero & 35 & 33 & 2 \\
Grado en Administración y & Cuarto & 13 & 8 & 5 \\
$\begin{array}{l}\text { Dirección de Empresas } \\
\text { Grado en Educación Infantil }\end{array}$ & Cuarto & 59 & 54 & 5 \\
Grado en Educación Primaria & Cuarto & 19 & 11 & 8 \\
Total & & 126 & 106 & 20 \\
\hline
\end{tabular}

Fuente: elaboración propia.

Para recopilar la percepción de los estudiantes, respecto al trabajo en equipo cooperativo como estrategia de trabajo que contribuye al desarrollo de su Competencia Social, se utilizó un cuestionario, elaborado ad hoc por los profesores investigadores participantes en el Proyecto de Innovación Docente. Así, plantea 41 preguntas para ser respondidas mediante una escala de valoración tipo Likert, con 5 opciones de respuesta y un intervalo de frecuencias donde 1 equivale a casi nunca/no se aprecia con claridad y 5 a casi siempre. El cuestionario requería señalar al encuestado con qué frecuencia considera que el Aprendizaje Cooperativo desarrolla capacidades, habilidades y competencias emergentes al desempeño de la Competencia Social: Empatía, Consenso o Participación Democrática y Asertividad. Su cumplimentación fue voluntaria, anónima. Aunque la población total objeto de estudio eran 264 estudiantes, finalmente cumplimenta el cuestionario un $47,7 \%$, que proyecta una muestra de 126 , planteando un nivel de confianza del $95 \%$, un $50 \%$ de heterogeneidad y un intervalo de confianza del 6,3. Su distribución se realizó digitalmente, desde las Aulas Virtuales correspondientes del Campus Virtual de la Institución Universitaria, insertando un link de acceso desde la sección Anuncios. La tecnología de soporte para los cuestionarios fue proporcionada por Google Formularios. 
Sánchez-Marín, F. J., Parra-Meroño, M. C., y Peña-Acuña, B. Experiencias de trabajo cooperativo en la educación superior. Percepciones sobre su contribución al desarrollo de la competencia social

Se comprobó la fiabilidad de las preguntas a partir del coeficiente de consistencia interna alfa de Cronbach, obteniendo un valor general de 0,963, considerado altamente fiable (Cohen, Manion y Morrison, 2013). Tanto las preguntas, como la estructura e ítems utilizados en el cuestionario fueron validados por expertos en disciplinas relacionadas con la temática objeto de estudio. Igualmente, se tuvieron en cuenta las publicaciones previas de los integrantes del equipo de investigación (Parra y Peña, 2012; Peña, Díez y García, 2012; Peña y Parra, 2011), así como autores y obras referentes en relación al marco teórico que sustenta el estudio en la materia tratada (Bisquerra y Pérez, 2007; Gardner, 2005; Goleman, 1995; Goleman, 2006; Salovey y Mayer, 1990). El tratamiento de la información recogida se realizó mediante análisis estadístico de datos cuantitativos basado en frecuencias, utilizando el paquete estadístico IBM SSPS Statistics 21.

\section{DISCUSIÓN}

Como se puede apreciar en la Figura 1, el resultado del análisis evidencia una percepción muy positiva acerca del grado en que consideran que el trabajo en equipo cooperativo contribuye a desarrollar la Competencia Social. Las puntuaciones se muestran muy similares en todas las capacidades: Empatía, Consenso o Participación Democrática y Asertividad.

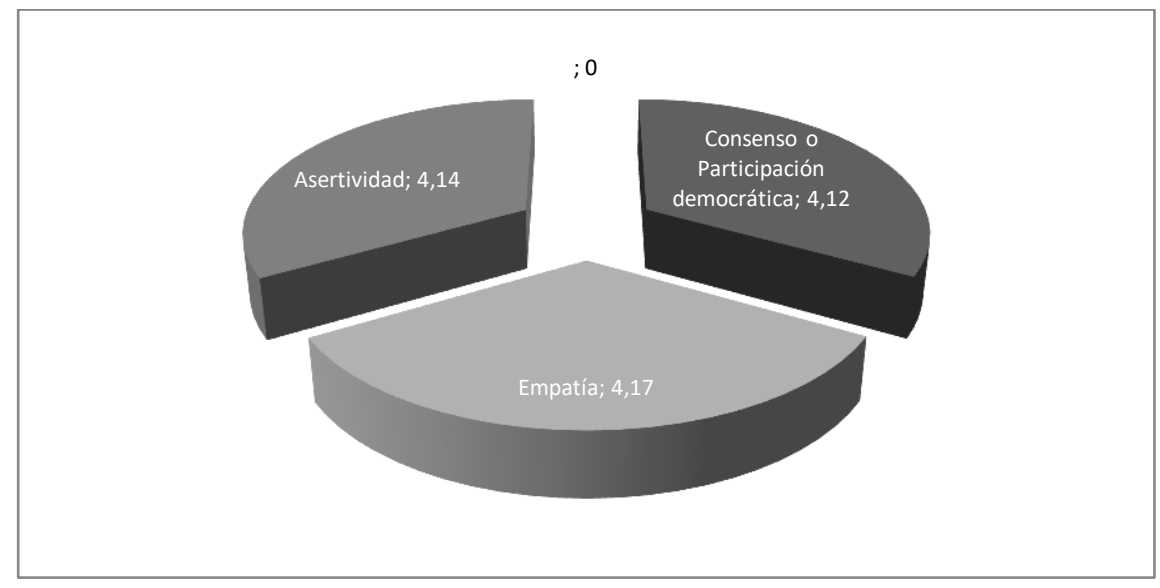

Figura 1. Puntuaciones globales de los estudiantes.

Fuente: elaboración propia.

La puntuación media total de los ítems incluidos en ellas (considerando una escala de puntuación del 1 al 5) se sitúan por encima del 4.1, con una desviación típica media de 0.9 .

En general, los estudiantes perciben que el Aprendizaje Cooperativo contribuye Muy a menudo (según su equivalencia con la escala nominal utilizada) a desarrollar la

Vivat Academia. Revista de Comunicación. 15 junio 2019 /15 septiembre 2019, nº 147, 87-108 
Sánchez-Marín, F. J., Parra-Meroño, M. C., y Peña-Acuña, B. Experiencias

de trabajo cooperativo en la educación superior. Percepciones sobre su contribución al desarrollo de la competencia social

Competencia Social. Entre ellas destacan aquellas relacionadas con la Empatía, con una puntuación de 4.17. Por el contrario, al conjunto de habilidades relacionadas con la capacidad de Consenso o Participación Democrática se les concede una menor puntuación, aunque la diferencia entre ambas capacidades no sea significativa; 4.12. La capacidad para la Asertividad se sitúa en una posición intermedia, con una puntuación media de 4.14. En adelante se exponen los resultados obtenidos para cada habilidad concreta que se recoge para cada capacidad estudiada.

\section{a) Capacidad de Empatía}

Esta capacidad incluye la habilidad mejor valorada de todas las estudiadas en esta investigación: Escuchar al otro, con una frecuencia de 4.6, lo que la acerca al valor nominal: Casi siempre. Esto supone que, más del 91\% de los estudiantes, la han puntuado con un 4 o con 5. En el lado opuesto encontramos, aunque con escasa diferencia: Utilizar lenguaje gestual para apoyar argumentos esgrimidos, con una puntuación de 4.02, más cercana al valor nominal: Muy a menudo. Esto representa un $70.6 \%$ de estudiantes que la puntúan con un 4 o 5 . La valoración que hacen los estudiantes acerca de la frecuencia con la que el Aprendizaje Cooperativo permite desarrollar el resto de habilidades asociadas a la Empatía, ordenadas de mayor a menor puntuación, se presenta en la Tabla 2.

Tabla 2. Contribución del aprendizaje cooperativo al desarrollo de habilidades asociadas a la empatía.

\begin{tabular}{lccc}
\hline \multicolumn{1}{c}{ Habilidad } & $\begin{array}{c}\text { Puntuación } \\
\text { Media }\end{array}$ & $\begin{array}{c}\text { Desviación } \\
\text { típica }\end{array}$ & $\begin{array}{c}\text { \% de } \\
\text { puntuaciones } \\
\text { entre 4 y 5 }\end{array}$ \\
\hline $\begin{array}{l}\text { Escuchar al otro } \\
\text { Analizar las diferentes opciones } \\
\text { propuestas por otros }\end{array}$ & 4.62 & 0.691 & 91.3 \\
$\begin{array}{l}\text { Aceptar y valorar otras opiniones o ideas } \\
\text { diferentes }\end{array}$ & 4.44 & 0.853 & 87.3 \\
$\begin{array}{l}\text { Sentir que se aprende y se abre la mente } \\
\text { escuchando otras soluciones }\end{array}$ & 4.33 & 0.808 & 83.3 \\
$\begin{array}{l}\text { Enriquecerse entendiendo otros puntos } \\
\text { de vista }\end{array}$ & 4.29 & 0.886 & 83.3 \\
$\begin{array}{l}\text { Ponerse en el lugar de los demás } \\
\text { Entender mejor otros modos de sentir y } \\
\text { emocionarse }\end{array}$ & 4.20 & 0.878 & 84.9 \\
$\begin{array}{l}\text { Evitar prejuicios } \\
\text { Utilizar el lenguaje gestual para apoyar } \\
\text { los argumentos }\end{array}$ & 4.11 & 0.867 & 81.0 \\
\hline
\end{tabular}

Fuente: elaboración propia. 
Sánchez-Marín, F. J., Parra-Meroño, M. C., y Peña-Acuña, B. Experiencias

de trabajo cooperativo en la educación superior. Percepciones sobre su contribución al desarrollo de la competencia social

\section{b) Capacidad para el Consenso o Participación Democrática}

La capacidad para el Consenso o Participación Democrática contiene la habilidad peor puntuada de todas por las que se interroga en esta investigación: Evitar contrarrestar argumentos, con una frecuencia de 3.7 que le atribuye el valor nominal: Con frecuencia. Esto significa que, el $65 \%$ de los estudiantes, le atribuye una puntuación entre 4 o 5 como tendencia general. Esto contrasta, con cierto grado de diferencia, con la habilidad: Reconocerse el mismo derecho que los demás a opinar y ser escuchado, a la que se le otorga una puntuación de 4.36, que la aproxima al valor nominal: Muy a menudo. Así, más del $86 \%$ de los estudiantes la puntúan con un 4 o 5 . Como se establecía para la capacidad de Empatía, la distribución de las estimaciones que hacen los estudiantes acerca de la frecuencia con la que el Aprendizaje Cooperativo permite desarrollar el resto de habilidades asociadas al Consenso o Participación Democrática, en orden ascendente, se muestra en la Tabla 3.

Tabla 3. Contribución del aprendizaje cooperativo al desarrollo de habilidades asociadas al consenso o participación democrática.

\begin{tabular}{lccc}
\hline \multicolumn{1}{c}{ Habilidad } & $\begin{array}{c}\text { Puntuación } \\
\text { Media }\end{array}$ & $\begin{array}{c}\text { Desviación } \\
\text { típica }\end{array}$ & $\begin{array}{c}\text { \% de } \\
\text { puntuaciones } \\
\text { entre 4 y 5 }\end{array}$ \\
\hline $\begin{array}{l}\text { Reconocerse el mismo derecho que los } \\
\text { demás a opinar y ser escuchado }\end{array}$ & 4.36 & 0.899 & 86.5 \\
$\begin{array}{l}\text { Sentir que se aprende más en equipo que } \\
\text { en solitario, en la convicción de que se } \\
\text { multiplica la percepción de la realidad }\end{array}$ & 4.32 & 0.836 & 82.5 \\
$\begin{array}{l}\text { Dejarse corregir, revisar por los demás, } \\
\text { admitir sugerencias de mejora }\end{array}$ & 4.29 & 0.849 & 84.9 \\
$\begin{array}{l}\text { Exponer las opiniones de manera } \\
\text { razonada }\end{array}$ & 4.25 & 0.809 & 81.7 \\
$\begin{array}{l}\text { Sentir la necesidad de conocer más a los } \\
\text { otros }\end{array}$ & 4.21 & 0.999 & 80.2 \\
$\begin{array}{l}\text { Comprender al otro y tener una buena } \\
\text { retroalimentación en las conversaciones }\end{array}$ & 4.21 & 0.864 & 81.7 \\
$\begin{array}{l}\text { Favorecer una interacción fluida } \\
\text { Respetar el turno de palabra, no }\end{array}$ & 4.19 & 0.817 & 81.7 \\
$\begin{array}{l}\text { interrumpir cuando otro habla } \\
\text { Satisfacer los intereses de todas las partes }\end{array}$ & 4.16 & 0.933 & 77.8 \\
$\begin{array}{l}\text { Utilizar un tono de voz bajo y calmado, } \\
\text { pese a creer que se tiene la razón, }\end{array}$ & 4.15 & 0.930 & 78.6 \\
$\begin{array}{l}\text { estando convencido que, de lo contrario, } \\
\text { la otra persona podría sentirse agredida } \\
\text { o cohibida }\end{array}$ & 4.15 & 1.036 & 76.2 \\
$\begin{array}{l}\text { Escuchar a los demás mirando a los ojos } \\
\text { estando convencido que así inspira } \\
\text { confianza y que realmente se está } \\
\text { comunicando }\end{array}$ & 4.12 & & \\
\hline
\end{tabular}

Vivat Academia. Revista de Comunicación. 15 junio 2019 /15 septiembre 2019, n 147, 87-108 
Sánchez-Marín, F. J., Parra-Meroño, M. C., y Peña-Acuña, B. Experiencias

de trabajo cooperativo en la educación superior. Percepciones sobre su contribución al desarrollo de la competencia social

\begin{tabular}{|c|c|c|c|}
\hline $\begin{array}{l}\text { Apreciar que todos tienen el mismo } \\
\text { poder y que nadie es dominante, ni se } \\
\text { sale con la suya }\end{array}$ & 4.07 & 1.089 & 75.4 \\
\hline Confiar unos en otros & 4.06 & 1.105 & 74.6 \\
\hline $\begin{array}{l}\text { Realizar concesiones: ceder ante posturas } \\
\text { u opiniones diferentes }\end{array}$ & 4.04 & 0.958 & 79.4 \\
\hline $\begin{array}{l}\text { Admitir el fracaso de equipo, a pesar de } \\
\text { creer que no se tiene la culpa } \\
\text { directamente }\end{array}$ & 3.99 & 1.008 & 69.8 \\
\hline Incentivar la participación de todos & 3.94 & 1.228 & 66.7 \\
\hline $\begin{array}{l}\text { Mostrar una actitud corporal de } \\
\text { apertura: relajada, tranquila y confiada }\end{array}$ & 3.91 & 0.988 & 69.8 \\
\hline Evitar contrarrestar argumentos & 3.75 & 1.035 & \\
\hline
\end{tabular}

Fuente: elaboración propia.

\section{c) Capacidad para la Asertividad}

Las habilidades relacionadas con la capacidad para la Asertividad mantienen una puntuación media muy parecida, no contienen ningún valor extremo dentro del cómputo global de las competencias estudiadas, como ocurre con las dos capacidades precedentes. No obstante, como se hizo anteriormente, se puede señalar, dentro de esta misma dimensión, como habilidad mejor apreciada: Defender el derecho a participar, sin atacar los derechos de los demás, con una frecuencia de 4.33, por lo que se asocia al valor nominal: Casi siempre. Quiere decir, entonces, que, algo más del $84 \%$ de los estudiantes, la han puntuado con un 4 o con un 5. La habilidad más alejada de esta puntuación es: Adoptar decisiones considerando previamente todas las opiniones y posturas al respecto, con una puntuación de 3.96, que la relaciona con el valor nominal: Con frecuencia. Es algo más del $70 \%$ de los estudiantes los que la puntúan con un 4 o 5 . El resto de habilidades afines a la capacidad de Asertividad se ordenan en la Tabla 4.

Tabla 4. Contribución del aprendizaje cooperativo al desarrollo de habilidades asociadas a la asertividad.

\begin{tabular}{lccc}
\hline \multicolumn{1}{c}{ Habilidad } & $\begin{array}{c}\text { Puntuación } \\
\text { Media }\end{array}$ & $\begin{array}{c}\text { Desviación } \\
\text { típica }\end{array}$ & $\begin{array}{c}\text { \% de } \\
\text { puntuaciones } \\
\text { entre 4 y 5 }\end{array}$ \\
\hline $\begin{array}{l}\text { Defender el derecho a participar, sin atacar } \\
\text { los derechos de los demás }\end{array}$ & 4.33 & 0.778 & 84.1 \\
$\begin{array}{l}\text { Participar con un estado emocional de } \\
\text { calma, evitando actitudes agresivas y } \\
\text { dominantes }\end{array}$ & 4.32 & 0.918 & 84.1 \\
$\begin{array}{l}\text { Comunicar mediante gestos suaves, no } \\
\text { invasivos }\end{array}$ & 4.25 & 0.876 & 81.7 \\
$\begin{array}{l}\text { Participar con naturalidad, no de forma } \\
\text { cohibida o pasiva por comodidad o por }\end{array}$ & 4.24 & 0.907 & 82.5 \\
\hline
\end{tabular}


Sánchez-Marín, F. J., Parra-Meroño, M. C., y Peña-Acuña, B. Experiencias

de trabajo cooperativo en la educación superior. Percepciones sobre su contribución al desarrollo de la competencia social

sentir que otros son más capaces o

dominantes

Mostrar una actitud comprensiva, no

sojuzgar (someter con violencia)

$\begin{array}{lll}4.23 & 1.029 & 81.0\end{array}$

Comprender lo que siente la otra persona

$\begin{array}{lll}4.13 & 0.915 & 78.6\end{array}$

Expresar sentimientos de acuerdo a las

decisiones adoptadas

$\begin{array}{lll}4.12 & 0.845 & 81.7\end{array}$

Defender la propia postura considerando el

impacto emocional que puede tener en el

4.10

0.875

79.4

otro

Evitar la adopción de posturas a la

defensiva

$\begin{array}{lll}4.08 & 1.032 & 71.4\end{array}$

Prever conflictos expresando de forma

tranquila lo que no gusta o no parece bien

4.06

0.901

74.6

Emplear un tono de voz relajado, sin

tensión

$\begin{array}{lll}4.06 & 0.919 & 73.0\end{array}$

Utilizar un ritmo de habla pausado y

tranquilo

$\begin{array}{lll}4.05 & 0.838 & 77.0\end{array}$

Hablar en primera persona cuando no se

representa a los demás para no repercutir

negativamente sobre la imagen del grupo

Adoptar decisiones considerando

previamente todas las opiniones y posturas

al respecto

$\begin{array}{lll}4.03 & 1.011 & 77.0\end{array}$

$\begin{array}{lll}3.96 & 0.975 & 70.6\end{array}$

Fuente: elaboración propia.

Los resultados obtenidos se pueden enmarcar en la línea de recientes investigaciones que subrayan el Aprendizaje Cooperativo en la Educación Superior como propuesta metodológica que reporta beneficios al estudiante y permite el desarrollo, entre otras cosas, de su Competencia Social (Lanza y Fernández, 2012). Esto también se demuestra en trabajos de tipo experimental realizados desde diversas disciplinas académicas, observándose mejoras significativas, en los respectivos grupos experimentales, en los niveles de percepción de competencia, motivación auto-determinada y relaciones sociales (Fernández-Río, Cecchini y Méndez-Giménez, 2014), así como en la autoconfianza y reducción de la ansiedad (Berger y Hanze, 2015; Kwok y Lau, 2015; Partridge y Eamoraphan, 2015). Ocurre igualmente en capacidades específicas incluidas en este estudio de tipo evaluativo como son la Empatía (Lirola, 2015; Rodríguez, Trianes y Casado, 2013), el Consenso o Participación Democrática y destrezas relacionadas con la Asertividad (Durán-Aponte y Durán-García, 2013; Huang, Wu y Hwang, 2015; Jauregi, Vidales, Casares y Fuente, 2014). Igualmente, las bondades del Aprendizaje Cooperativo están avaladas con los últimos hallazgos de la neurociencia social (Clark y Dumas, 2015; Necka, Cacioppo y Cacioppo, 2015).

Este estudio de tipo evaluativo aporta novedad en tanto que explora sobre una muestra de estudiantes con perfiles diversos, lo hace tomando como punto de partida de

Vivat Academia. Revista de Comunicación. 15 junio 2019 /15 septiembre 2019, nº 147, 87-108 
Sánchez-Marín, F. J., Parra-Meroño, M. C., y Peña-Acuña, B. Experiencias

de trabajo cooperativo en la educación superior. Percepciones sobre su contribución al desarrollo de la competencia social

manera integrada las variables Asertividad, Consenso o Participación democrática y Empatía y sobre la percepción que los propios estudiantes expresan de manera explícita. De esta forma se confirma que los estudiantes perciben que, a través del Aprendizaje Cooperativo, se desarrolla su Competencia Social y, por tanto, se asume que valoran muy positivamente este tipo de estrategia metodológica para lograrlo. Las habilidades mejor valoradas son las que se relacionan con el ámbito cognitivo y se relega a un segundo, aunque con escasa diferencia, otras destrezas de carácter emocional, axiológico y paradigmático relacionadas con la alteridad, el valor de justicia, el compartir y el reconocimiento al derecho de participación. Escuchar al otro, reconocer el mismo derecho que los demás a participar, opinar y ser escuchado, se convierten en las destrezas mejor valoradas por los estudiantes con una orientación cognitiva, en detrimento de otras más relacionadas con el ámbito emocional; lenguaje corporal, actitudinal y paradigmática: utilizar el lenguaje gestual para apoyar los argumentos esgrimidos, evitar contrarrestar argumentos y adoptar decisiones considerando previamente todas las opiniones y posturas al respecto.

\section{CONCLUSIONES}

Después de realizar una observación participante durante años (2009-2013), realizar una investigación-acción participativa en un curso académico (2013-2014) y realizar un proyecto de innovación educativa sobre el trabajo cooperativo, estimamos que la investigación cualitativa que combina varias técnicas y herramientas durante años en la Educación superior sobre un mismo tema resulta muy eficaz, así como asegura la adecuación a la diversidad del alumnado.

Queremos resaltar en este caso, la vertebración de la acción participativa en el proyecto piloto para crear un proyecto de innovación educativa con más envergadura como muy adecuada para profundizar en la realidad y dotar de variables de punto de partida tanto para el proyecto de innovación, como para un proyecto de investigación. Las ventajas de la investigación-acción participativa que enumeramos son muchas. Primero, dota al profesor de herramientas de investigación y mejora docente porque profundiza, lo adecua a la realidad del aula que él guía y le permite entender las necesidades de los alumnos. Segundo, es un medio humano favoreciendo un clima que permite al alumno expresarse sobre su proceso de aprendizaje, cuestión que le estimula y además favorece la extroversión con sus compañeros, así como otros factores como son la gestión de conflictos, el mayor conocimiento mutuo y la comprensión. Tercero, facilita que el alumno entienda la investigación del profesorado en Educación, tanto del proyecto como de las técnicas utilizadas, así como de la actitud de humildad del profesor que busca entender al alumnado para desplegar metodologías docentes adecuadas.

Con respecto a los resultados de la investigación cualitativa y del proyecto de innovación, finalmente podemos concluir con que resulta pertinente replicar la 
Sánchez-Marín, F. J., Parra-Meroño, M. C., y Peña-Acuña, B. Experiencias de trabajo cooperativo en la educación superior. Percepciones sobre su contribución al desarrollo de la competencia social

experiencia y continuar con la utilización del Aprendizaje Cooperativo. Por otro lado, las medidas que podrían contrarrestar el impacto de las posibles limitaciones de este estudio de tipo evaluativo sobre los resultados obtenidos son: Incrementar el tamaño de la muestra para poder generalizar en mayor medida los resultados obtenidos, aumentar la duración del estudio para valorar el grado de desgaste en los agentes participantes por el cansancio generado por la dinámica de trabajo en períodos más largos de intervención que el utilizado y examinar los factores que pudieran dificultar la participación de los estudiantes en el Aprendizaje Cooperativo (Lanza, 2015; Shimazoe y Aldrich, 2010).

Los resultados obtenidos nos animan a continuar con la línea de investigación iniciada a la vez que plantean nuevos interrogantes. Algunas líneas de trabajo futuras son: ampliar los contextos de implementación del Aprendizaje Cooperativo y analizar las diferencias en sus resultados. Incluir las estrategias de aprendizaje cooperativo en asignaturas hasta el momento orientadas a la clase magistral y valorar diferencias en los resultados sobre la idoneidad de la metodología para aulas de clase más grandes y orientadas a las clases magistrales, adaptar la metodología del Aprendizaje Cooperativo para incorporarlo a la modalidad de comunicación a través de las TIC (Salmerón, Rodríguez y Gutiérrez, 2010) e incluir en las estrategias utilizadas, los hallazgos de las neurociencias sociales relacionados con la competencia social; empatía y conductas prosociales (Necka y otros, 2015).

\section{REFERENCIAS}

Aguaded, I. (2013). El Programa «Media» de la Comisión Europea, apoyo internacional a la educación en medios [Media Programme (EU) - International Support for Media Education]. Comunicar, 40(20), 7-8. doi: http://dx.doi.org/10.3916/C40-2013-01-01

Arnaiz, P. y Linares, J. E. (2010). Proyecto ACOOP. Murcia: Universidad de Murcia y Consejería de Educación, Formación y Empleo.

Berger, R. y Hanze, M. (2015). Impact of expert teaching quality on novice academic performance in the jigsaw cooperative learning method. International Journal of Science Education, 37(2), 294-320. doi: 10.1080/09500693.2014.985757

Bisquerra, R. y Escoda, N. P. (2007). Las competencias emocionales, Educación XX1, 10, 61-82. doi: 10.5944/educxx1.1.10.297

Camilli, C. (2015). Aprendizaje cooperativo e individual en el rendimiento académico en estudiantes universitarios: un meta-análisis, (Tesis Doctoral, Universidad Complutense de Madrid). Disponible en http://eprints.ucm.es/30997/ 
Sánchez-Marín, F. J., Parra-Meroño, M. C., y Peña-Acuña, B. Experiencias de trabajo cooperativo en la educación superior. Percepciones sobre su contribución al desarrollo de la competencia social

Cano, M. E. (2008). La evaluación por competencias en la educación superior. Profesorado. Revista de currículum y formación del profesorado, 12(3), 1-16.

Cebrián-De La Serna, M., Serrano-Angulo, J., y Ruiz-Torres, M. (2014). Las eRúbricas en la evaluación cooperativa del aprendizaje en la Universidad [eRubrics in Cooperative Assessment of Learning at University]. Comunicar, 43(22), 153-161. doi: http://dx.doi.org/10.3916/C43-2014-15

Clark, I. y Dumas, G. (2015). Toward a neural basis for peer-interaction: what makes peer-learning tick? Frontiers in psychology, 6, 1-12. doi: 10.3389/fpsyg.2015.00028

Cohen, L., Manion, L. y Morrison, K. (2013). Research methods in education. London y New York: Routledge.

Decety, J., Norman, G. J., Berntson, G. G. y Cacioppo, J. T. (2012). A neurobehavioral evolutionary perspective on the mechanisms underlying empathy. Progress in neurobiology, 98(1), 38-48. doi: 10.1016/j.pneurobio.2012.05.001

Decety, J., Bartal, I. B. A., Uzefovsky, F. y Knafo-Noam, A. (2016). Empathy as a driver of prosocial behaviour: highly conserved neurobehavioural mechanisms across species. Phil. Trans. R. Soc., 371(1686), 1-11. doi: http://dx.doi.org/10.1098/rstb.2015.0077

Del Barco, B. L., Felipe, E., Mendo, S. y Iglesias, D. (2015). Habilidades sociales en equipos de aprendizaje cooperativo en el contexto universitario, Psicología conductual. Revista internacional de psicología clínica y de la salud, 23(2), 191-214.

Del Barco, B. L., Mendo, S., Felipe, E., Polo, M. I. y Fajardo, F. (2015). Team Potency and Cooperative Learning in the University Setting. Journal of Psychodidactics, 21(2), 1-15. doi: http://dx.doi.org/10.1387/RevPsicodidact.14213

Durán-Aponte, E. y Durán-García, M. (2013). Aprendizaje cooperativo en la Enseñanza de Termodinámica: Estilos de Aprendizaje y Atribuciones Causales. Journal of Learning Styles, 6(11), 256-275.

European Commission/Eacea/Eurydice (2015). The European Higher Education Area in 2015: Bologna Process Implementation Report. Luxembourg: Publications Office of the European Union. doi: 10.2797/128576

Fernández-Río, J., Cecchini, J.A. y Méndez-Giménez, A. (2014). Effects of cooperative learning on perceived competence, motivation, social goals, effort and boredom in 
Sánchez-Marín, F. J., Parra-Meroño, M. C., y Peña-Acuña, B. Experiencias de trabajo cooperativo en la educación superior. Percepciones sobre su contribución al desarrollo de la competencia social

prospective Primary Education teachers, Infancia y Aprendizaje: Journal for the Study of Education and Development, 37(1), 57-89. doi: 10.1080/02103702.2014.881650

Ferrés, J. (2007). La competencia en comunicación audiovisual: dimensiones e indicadores, Comunicar, 29(15), 100-107.

García, A. y Troyano, Y. (2010). Aprendizaje cooperativo en personas mayores universitarias. Revista Interamericana de Educación de Adultos, 32(1), 7-21.

Gardner, H. (2005). Inteligencias múltiples. La teoría en la práctica. Barcelona: Paidós.

Gast, D. L. y Ledford, J. R. (2014). Applied research in education and behavioral sciences. Single Case Research Methodology: Applications in Special Education and Behavioral Sciences. New York y London: Routledge.

Gil, P. (2015). Percepciones hacia el aprendizaje cooperativo del alumnado del Máster de Formación del Profesorado de Secundaria. REDU. Revista de Docencia Universitaria, 13(3), 125-146.

Goleman, D. (1995). Emotional Intelligence. New York: Bantam Books.

Goleman, D. (2006). Inteligencia social: la nueva ciencia de las relaciones humanas. Barcelona: Kairós.

Huang, Y. H., Wu, P. H. y Hwang, G. J. (2015, July). The Pilot Study of the Cooperative Learning Interactive Model in e-Classroom towards Students' Learning Behaviors". In T. Matsuo, K. Hashimoto, T. Mine y S. Hirokawa (Eds.), Advanced Applied Informatics (IIAI-AAI), 2015 IIAI 4th International Congress on. Okayama: CPS, pp. 279282. doi: 10.1109/IIAI-AAI.2015.193

Jauregi, P. A., Vidales, K. B., Casares, S. M. G. y Fuente, A.V. (2014). Estudio de caso y aprendizaje cooperativo en la universidad. Profesorado: Revista de curriculum y formación del profesorado, 18(1), 413-429.

Johnson, D. W., Johnson, R. T. y Holubec, E. J. (1999). El aprendizaje cooperativo en el aula. Barcelona: Paidós.

Jurkowski, S. y Hanze, M. (2015). How to increase the benefits of cooperation: Effects of training in transactive communication on cooperative learning, British Journal of Educational Psychology, 85(3), 357-371. doi: 10.1111/bjep.12077 
Sánchez-Marín, F. J., Parra-Meroño, M. C., y Peña-Acuña, B. Experiencias de trabajo cooperativo en la educación superior. Percepciones sobre su contribución al desarrollo de la competencia social

Kwok, A. P. y Lau, A. (2015). An Exploratory Study on Using the Think-Pair-Share Cooperative Learning Strategy. Journal of Mathematical Sciences, 2, 22-28.

Lanza, D. y Fernández, A. B. (2012). Aprendizaje cooperativo como fórmula para el desarrollo de competencias en el Espacio Europeo de Educación Superior: un estudio exploratorio con alumnos de Psicología de la Universidad Autónoma de Madrid, Revista del Congrés Internacional de Docència Universitària $i$ Innovació (CIDUI), 1(1).

Lanza, D. (2015). Aprendizaje cooperativo en el espacio europeo de educación superior: debilidades y fortalezas de una metodología didáctica, Revista del Congrés Internacional de Docència Universitària i Innovació (CIDUI), 2, 1-14.

Lirola, M. M. (2015). The Potential of Cooperative Learning and Peace Education to Promote the Acquisition of Social Competences at Tertiary Education, International Journal of Peace, Education and Development, 3(1), 37-47.

Mertens, D. M. (2014). Research and Evaluation in Education and Psychology: Integrating Diversity with Quantitative, Qualitative, and Mixed Methods. Thousands Oaks: Sage Publications.

Mulder, M., Weigel, T. y Collings, K. (2008). El concepto de competencia en el desarrollo de la educación y formación profesional en determinados países, miembros de la UE. Profesorado: Revista de curriculum y formación del profesorado, 12(3), 1-26.

Necka, E. A., Cacioppo, S. y Cacioppo, J. T. (2015). Social Neuroscience of the TwentyFirst Century, International Encyclopedia of the Social and Behavioral Sciences, 2(22), 485-488. doi: http://dx.doi.org/10.1016/B978-0-08-097086-8.56020-6

Oricain, N. D. (2015). Adquisición de competencias en el marco del Aprendizaje Cooperativo: valoración de los estudiantes, REDU: Revista de Docencia Universitaria, 13(1), 339-359.

Ortiz, M., Vicedo, A., González, S. y Recino, U. (2015). Las múltiples definiciones del término «competencia» y la aplicabilidad de su enfoque en ciencias médicas, Edumecentro, 7(3), 20-31.

Parra, M. C. y Peña, B. (2012). El aprendizaje cooperativo mediante actividades participativas, Revista Anales de la Universidad Metropolitana, 12(2), 15-37. 
Sánchez-Marín, F. J., Parra-Meroño, M. C., y Peña-Acuña, B. Experiencias de trabajo cooperativo en la educación superior. Percepciones sobre su contribución al desarrollo de la competencia social

Partridge, B. J. y Eamoraphan, S. (2015). A comparative study on students'foreign language classroom anxiety through cooperative learning on grade 10 students at Saint Joseph Bangna School, Thailand. Scholar, 7(1), 171-185.

Peña, B. y Parra, M. C. (2011). Aprendizaje cooperativo en observación sistemática mediante el visionado de films. Revista Vivat Academia, 117E, pp. 701-712. doi: http://dx.doi.org/10.15178/va.2011.117E.701-712

Peña, B., Díez, M. y García, M. (2012). La conveniencia de la inteligencia emocional por parte de los maestros de infantil y primaria En B. Peña (Coord.), Desarrollo humano, 15-48. Madrid: Visión libros.

Peña, B. (2014). Evaluación mediante la observación participante de una metodología de trabajo en equipo para el desarrollo de competencias básicas de inteligencia social. En González J. J., Sánchez, F. J. y Castejón M. A. Innovación Evaluación y Universidad, 46-60. Murcia: UCAM.

Rabadán, J. A. y Pérez, E. (2012). Renovación pedagógica en la sociedad del conocimiento, Nuevos retos para el profesorado universitario. RED-DUSC. Revista de Educación a Distancia-Docencia Universitaria en la Sociedad del Conocimiento, (6), 1-11.

Restrepo, J. A. (2014). La argumentación de las competencias en educación superior, ¿conducen a emulación o a generar virtud? No siempre el saber hacer implica el saber ser. Revista Interamericana de Investigación, Educación y Pedagogía, RIIEP, 7(2), 235-250.

Rodríguez, F. M. M., Trianes, M. V. y Casado, A. M. (2013). Eficacia de un programa para fomentar la adquisición de competencias solidarias en estudiantes universitarios, European Journal of Education and Psychology, 6(2), 95-104.

Román, S. S., Vecina, C., Usategui, E., Del Valle, A. I. y Venegas, M. (2015). Social representations and educational guidance of the professorship, Education Policy Analysis Archives, 23, 1-18. doi: http://dx.doi.org/10.14507/epaa.v23.2088

Salovey, P. y Mayer, J.D. (1990). Emotional intelligence, Imagination, cognition and personality, 9(3), 185-211.

Salmerón, H., Rodríguez, S. y Gutiérrez, C. (2010). Metodologías que optimizan la comunicación en entornos de aprendizaje virtual, Comunicar, 34(17), 163-171. doi: 10.3916/C34-2010-03-16 
Sánchez-Marín, F. J., Parra-Meroño, M. C., y Peña-Acuña, B. Experiencias de trabajo cooperativo en la educación superior. Percepciones sobre su contribución al desarrollo de la competencia social

Shimazoe, J. y Aldrich, H. (2010). Group work can be gratifying: Understanding and overcoming resistance to cooperative learning, College Teaching, 58, 52-57.

Más, O. y Olmos, P. (2012). La atención a la diversidad en la educación superior: una perspectiva desde las competencias docentes, Revista de Educación Inclusiva, 5(1), 159174.

Trujillo, A. L. y Sánchez, M. T. (2013). Apuntes sobre la internacionalización y la globalización en educación: de la internacionalización de los modelos educativos a un nuevo modelo de gobernanza, Journal of Supranational Policies of Education, (1), 5366.

\section{AUTORES}

\section{Francisco José Sánchez Marín}

Doctor en Pedagogía y Máster en Derecho Sanitario y Bioética. Profesor Contratado Doctor en la Universidad Católica San Antonio de Murcia (UCAM). Ha participado en proyectos relacionados con la Educación y Tecnologías de la Información y Comunicación, Diversidad, Inclusión, Trabajo Autónomo del estudiante, Competencias digitales y Buenas prácticas en el marco del EEES, Innovación en metodología docente y evaluación de aprendizaje. Sus líneas de investigación abarcan: valores y educación, escuela inclusiva, organización y gestión escolar, formación continuada del profesorado, tecnologías aplicadas a la educación, atención a la diversidad y métodos educativos. fjsanchez235@ucam.edu

Orcid ID: http:// orcid.org/0000-0003-3164-6333

Google Scholar: https://goo.gl/8HQETY

ResearchID: http://www.researcherid.com/rid/L-8164-2014

ResearchGate: https://www.researchgate.net/profile/Francisco_Sanchez_Marin

\section{María Concepción Parra Meroño}

Doctora en Administración y Dirección de empresas: Marketing y Organización. Es profesora en el Grado en ADE y en el Master en Marketing y Comunicación y en el Master MBA de la UCAM. Sus principales líneas de investigación son la innovación educativa el comportamiento del consumidor, el marketing y la comunicación. Ha publicado en revistas de impacto cómo Estudios Sobre Educación, Historia y Comunicación Social, Revista Opción, Cuadernos de Economía y Dirección de la Empresa, Journal of Knowledge Management. Ha publicado en editoriales de prestigio (ranking SPI) como Tecnos, McGrawHill, UOC o Pirámide.

mcparra@ucam.edu

Orcid ID: http:// orcid.org/0000-0002-0457-4613

Google Scholar: https://scholar.google.es/citations?user=H49mMdgAAAAJ\&hl=es 
Sánchez-Marín, F. J., Parra-Meroño, M. C., y Peña-Acuña, B. Experiencias

de trabajo cooperativo en la educación superior. Percepciones sobre su contribución al desarrollo de la competencia social

ResearchGate: https://www.researchgate.net/profile/Maria_Concepcion_Parra

\section{Beatriz Peña Acuña}

Ha participado en congresos nacionales e internacionales y ha publicado en revistas de impacto, así como en editoriales de prestigio. Profesora Titular acreditada. Premio extraordinario de doctorado. Investigador principal del grupo Desarrollo personal. Sus principales líneas de investigación son la innovación educativa y la comunicación. Ha publicado en revistas de impacto cómo Estudios Sobre Educación, Historia y Comunicación Social, Chasqui, Revista Opción y libros en editoriales en ranking SPI como Tecnos, McGrawHill, Dykinson, Cambridge scholar publishing, MediaXXI. bpena@ucam.edu

Orcid ID: http:/ / orcid.org/0000-0003-2577-5681

Google Scholar: https://scholar.google.es/citations?user=-yTRm0cAAAAJ\&hl=es

ResearchID: http://www.researcherid.com/rid/L-9229-2014

ResearchGate: https://www.researchgate.net/profile/Beatriz_Pena-Acuna

Academia.edu: http:// ucam.academia.edu/BeatrizPe\%C3\%B1aAcu\%C3\%B1a

Mendeley: https://www.mendeley.com/profiles/beatriz-pena-acuna/ 